
where $L$ is the effective length of a magnet and the sum is taken over all the magnets around the ring. For a given value of $\mathrm{B}_{0}$ the momentum of the orbit is given by:

$$
p=e_{0} L / \theta
$$

We shall call this orbit the Optimum Central Orbit (OCO). It is a unique orbit. The machine is designed so that when this relationship exists between $B$ and $p$ there is only one central orbit and it is this smooth simple one. Off momentum orbits display oscillatory behavior and are symmetrically displaced from the central orbit. From TN 215 we know that in the "BEAM PROGRAM" coordinate system this orbit is displaced toward the center of the ring by 0.191 inches in straight sections between long magnets and 0.131 inches in straight sections between short magnets. In transition sections it is the mean of these.

In order to demonstrate this orbit we go to EDC-53 (Where is Zero? E.D. Courant, November 21, 1963). Figure 2 shows orbits 4 and 5 from that paper plotted in the BEAM coordinate system for the intermediate field case $\left(B_{0}=4965\right.$ Gauss $)$. They have momentum values of 16.67 and 16.75 Megagauss inches. In TN 215 the magnetic fringe field added 4 inches to the magnet length; in EDC-53 it adds 4.1 inches. This has inconsequential effects on the geometry but we must keep track of it. For EDC-53 the bend angle in a long magnet is:

$$
\begin{aligned}
\theta & =2 \pi \times 94.1 /(144 \times 94.1+96 \times 79.1) \\
& =27.963 \mathrm{mr}
\end{aligned}
$$

and the required momentum is:

$$
\begin{aligned}
p & =e B_{0} L / \theta \\
& =16.708 \text { megagauss inches }
\end{aligned}
$$


(See also EDC-37, Location of the Nominal Equilibrium Orbit in AGS, E.D. Courant, January 14, 1960, which comes to the same results as the present note). This momentum is very conveniently just the mean of orbits 4 and 5. Figure 3 shows orbits 4 and 5 and their mean, which clearly corresponds to the optimum central orbit we have been seeking. Figure 4 transforms these three orbits into the OCO coordinate system and we see a very nice symmetry appear for the off momentum orbits while the central orbit is absolutely flat in this coordinate system. Figure 5 shows orbits 2, 4, 5, and 7 from EDC-53 and the symmetry clearly persists. In this coordinate system we can easily write down a momentum dispersion function which is just the orbit displacement divided by the percentage momentum shift. Figure 6 shows the dispersion function and Figure 7 shows the dispersion function and a scaled value of the beta function (as tabulated in EDC-28).

III. Conclusions

The AGS as originally designed is conceptually a very simple machine. The optimum central orbit goes around the ring just as though it were going through a string of dipole magnets. The off momentum orbits and the beta function behave as though they were seeing a continuous string of uniform quadrupole doublets. This was the intent of the original machine designers. Many drawings still show a coordinate identified as "Theoretical Beam Center Line" or as " $\mathrm{R}_{0}$ " This coordinate is identical with what we have called the "OCO" coordinate system. The AGS standards book clearly specifies that the trim quadrupole and sextupole magnets are to be located on the oco center line. TN 212 (Maximum Limiting Aperture in the AGS, K.A. Brown and K.M. Brown, April 12, 1985) shows that the old pick up electrodes and many of the vacuum chambers are centered on the OCO system. However, the new pickup electrodes are thought to be centered on the "BEAM" program coordinate system (L. Ahrens, private communication). Thus we may be using a system that is not optimal conceptually, and we may be using two different systems unknowingly (J.P. Potier, private communication). 
IV. Recommendations

1. Choose one coordinate system. The OCO system is conceptually the most desirable.

2. Most groups, such as the control room, device designers, mechanical engineers, and design room, will use only that system.

3. For the surveyors prepare one drawing relating the oco system to the socket system.

4. For tracking program users prepare one drawing relating the OCO system to the "BEAM" program coordinate system.

5. Require that the output of all tracking progams be in the OCO coordinate system.

6. Establish the coordinates of all the ring devices in the OCO system.

7. All future devices and drawings should be prepared in the oco system.

8. Consider presenting device information to the control room in the OCO system. 
Table 1

MAGNET PARAMETERS

Long Magnet Short Magnet Units

Magnet Length
Magnet Effective Length
$Y_{0}$
$\theta / 2=\tan ^{-1}\left(\mathrm{Y}_{0}^{\prime}\right)$

90.000

75.000

Inches

94.000

79.000

Inches

0.21906

0.15472 Inches

13.9825

11.7512

$\mathrm{mr}$

Straight Section Lengths

2 foot

5 foot

10 foot
28.000

64.000

123.9907
Inches

Inches

Inches 


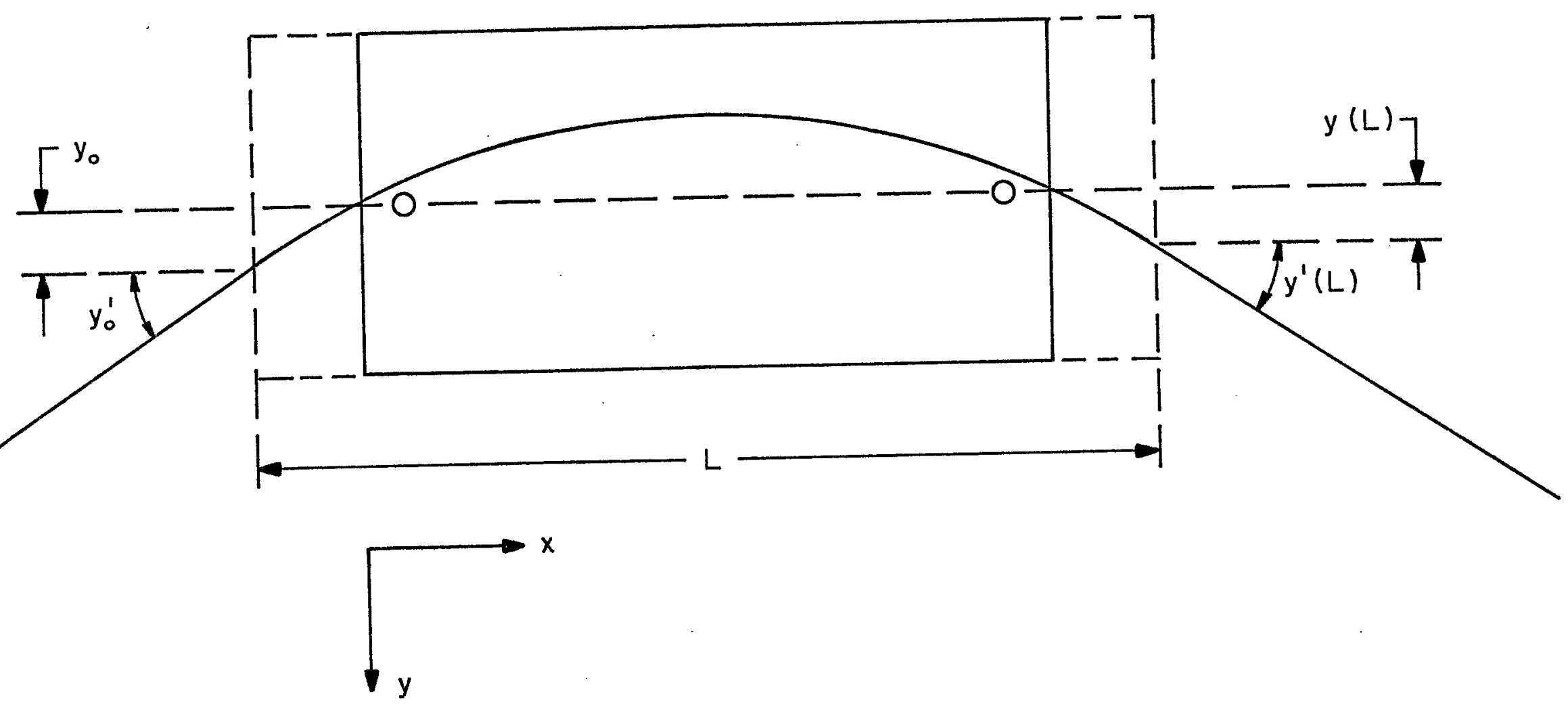

Figure 1 


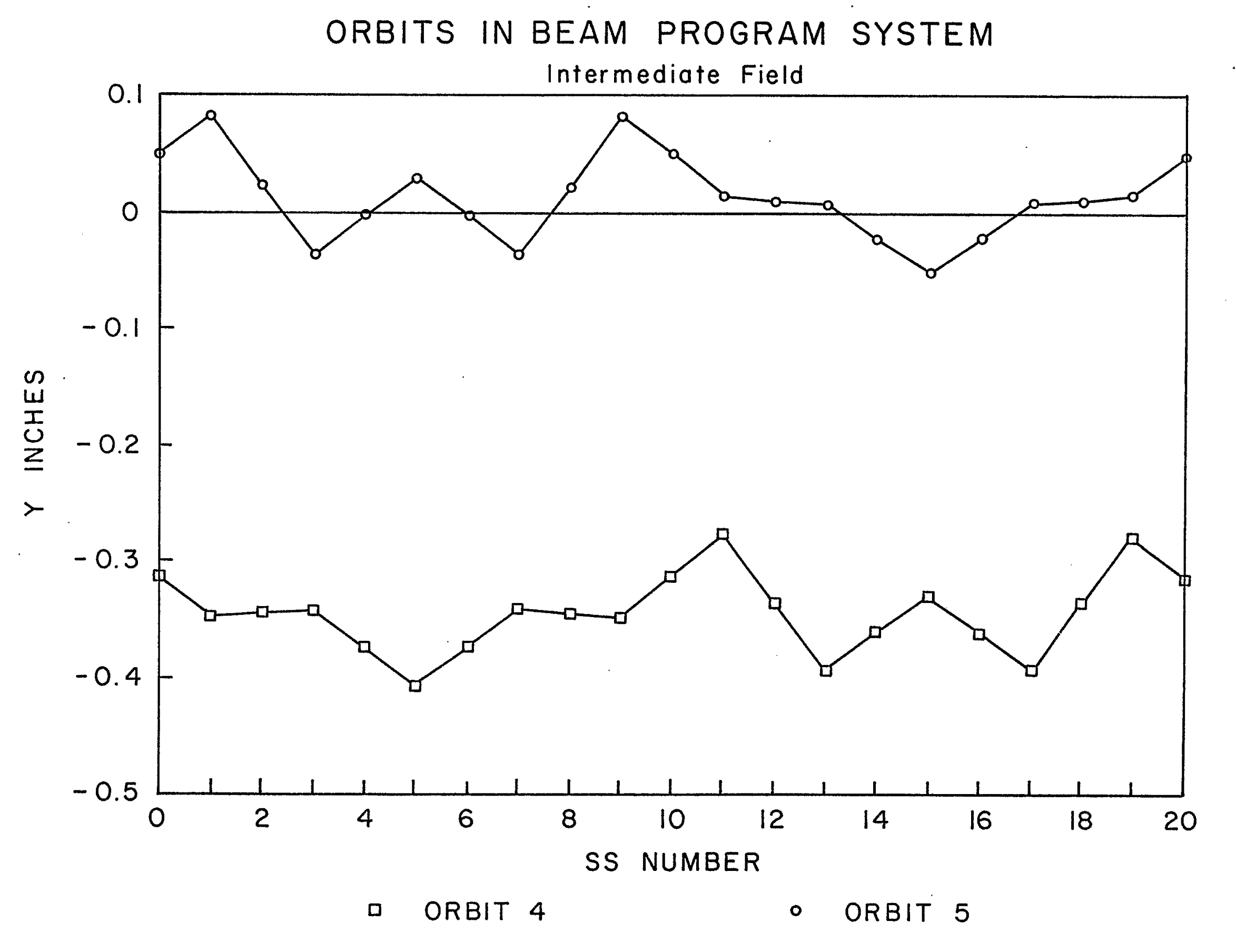

Figure 2 
ORBITS IN BEAM PROGRAM SYSTEM

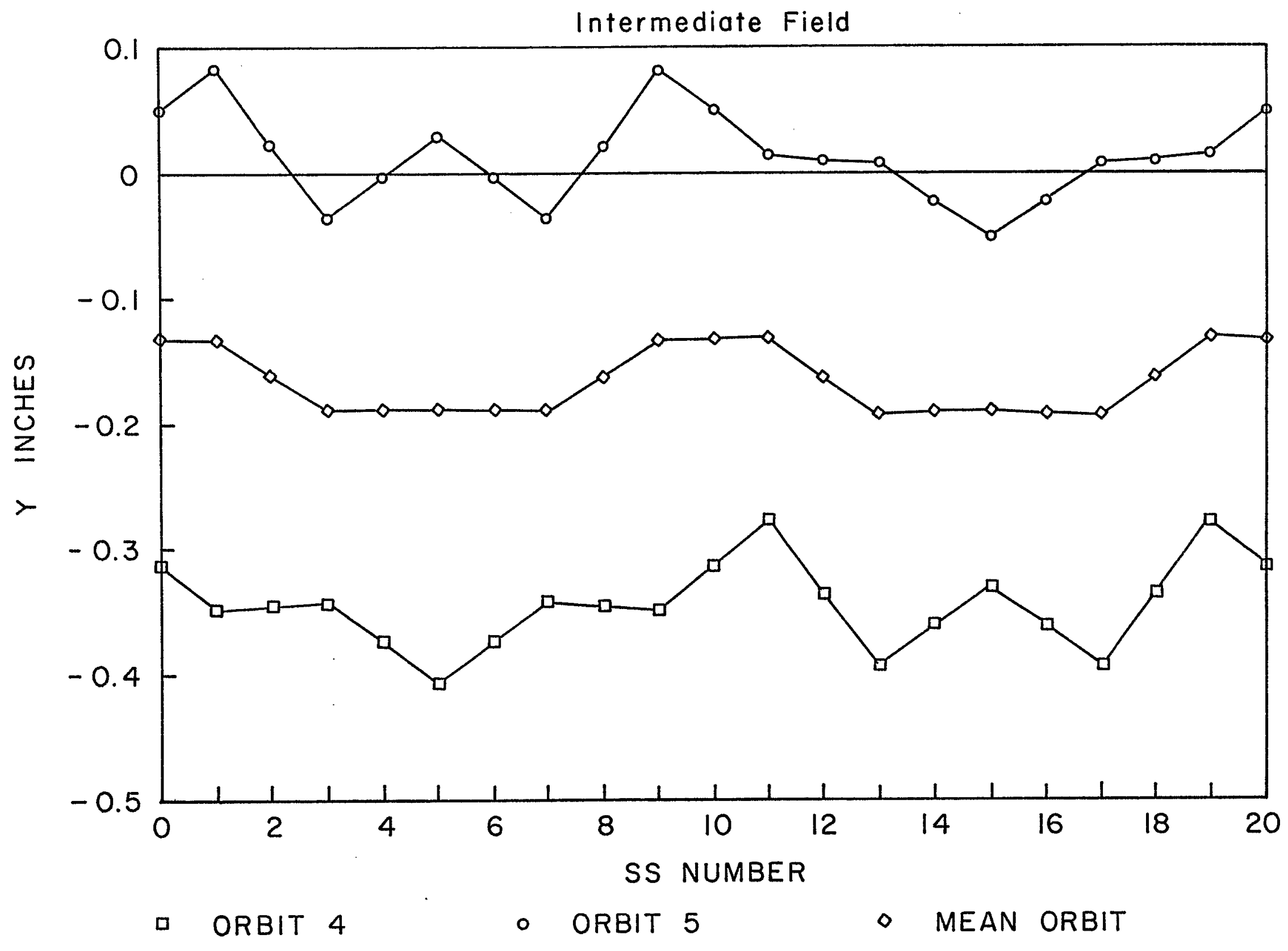




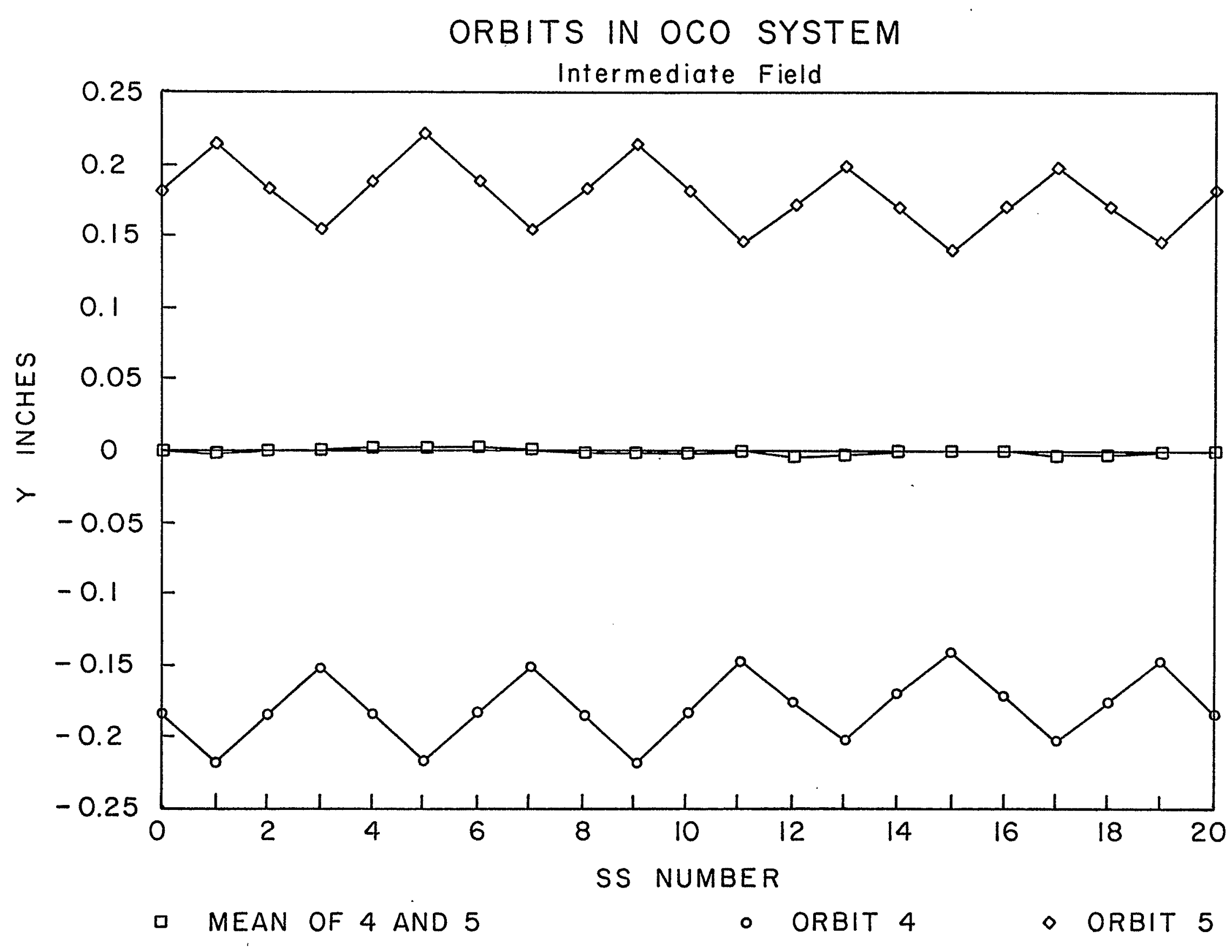




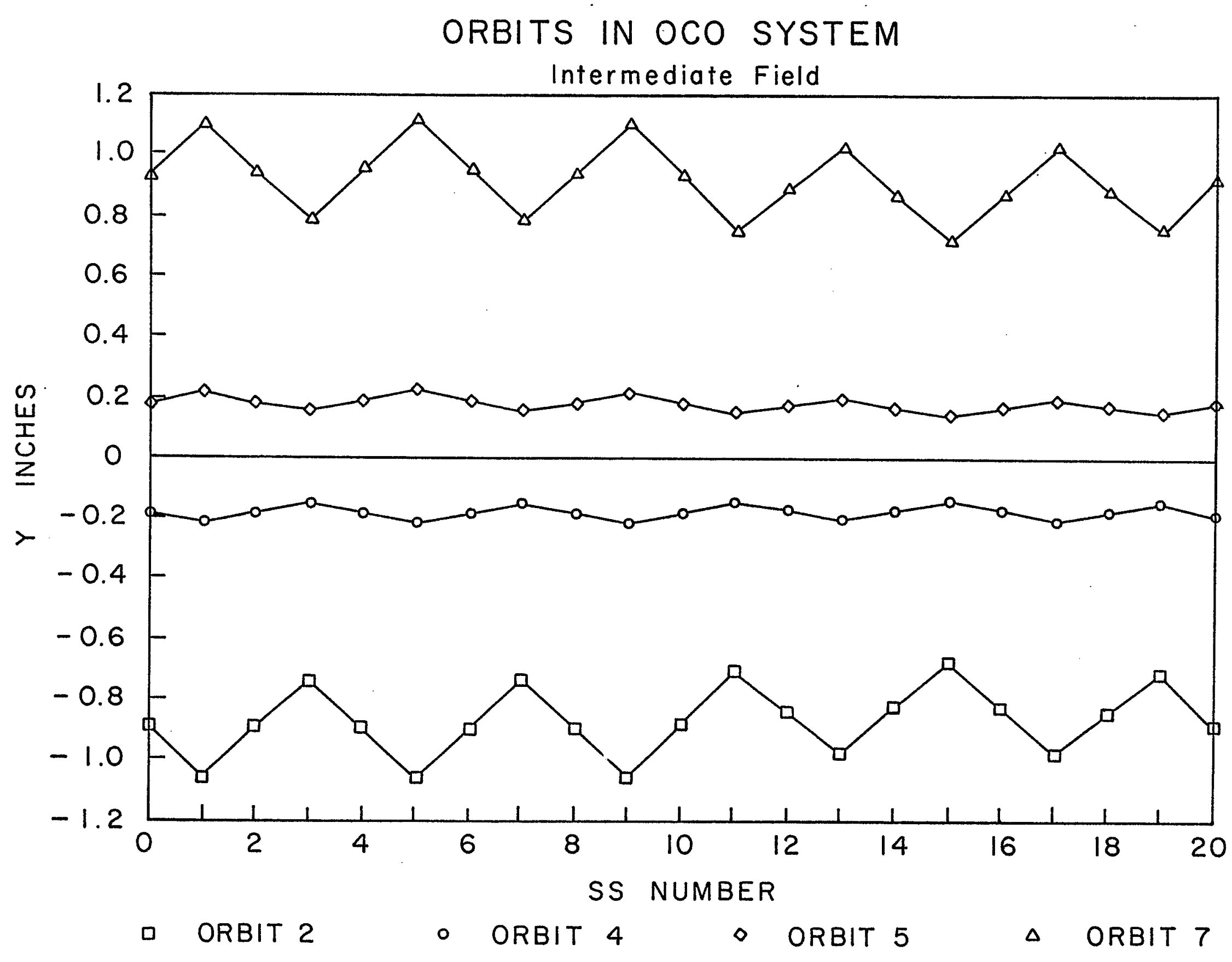

Figure 5 


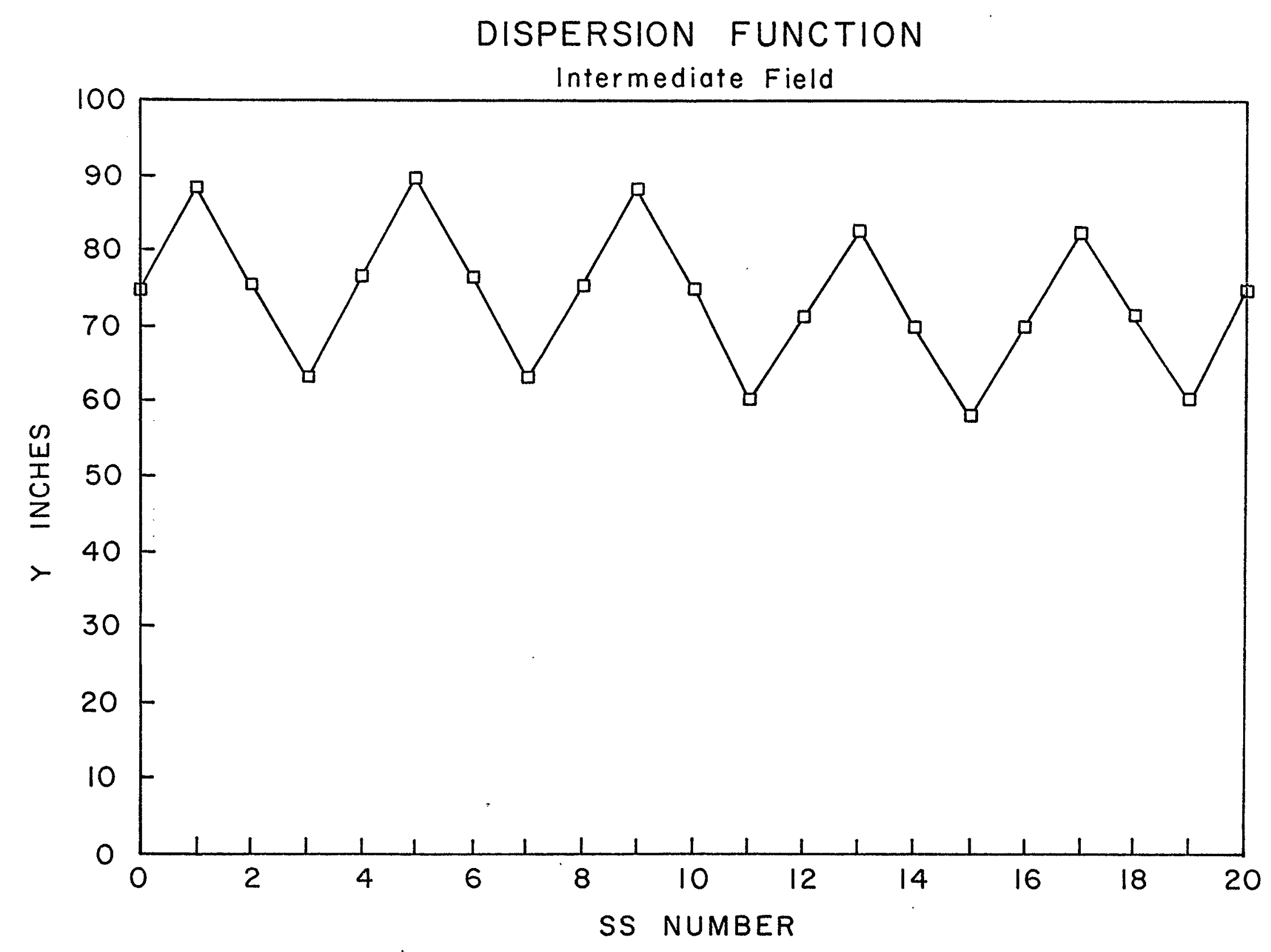

- ORBIT $7 / 0.0125$ 


\section{DISPERSION FUNCTION AND BETA FUNCTION}

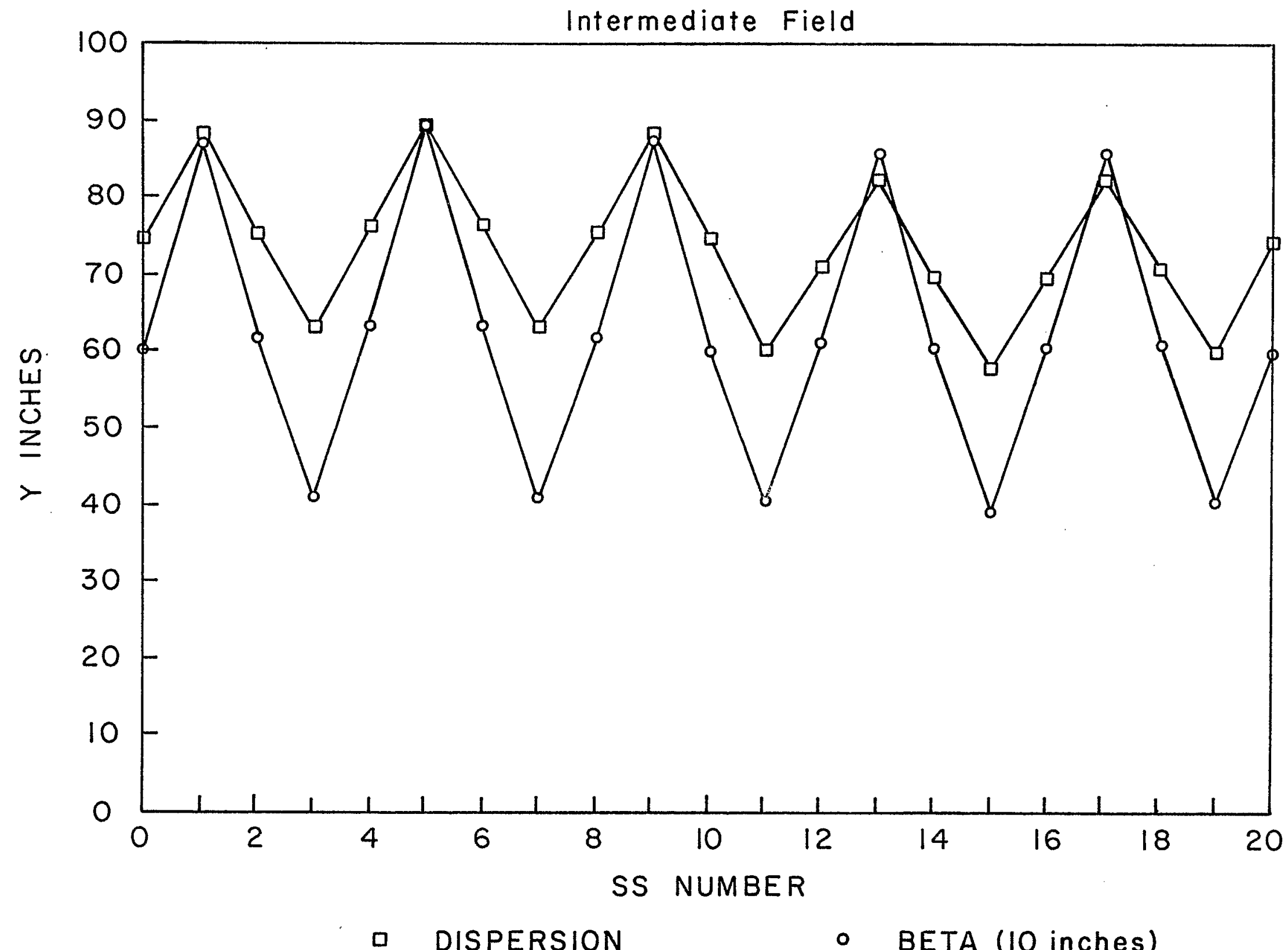


APPENDIX I

THE OCO COORDINATE SYSTEM

Figure Al attempts to make clear the differences between the various systems being discussed here. The "BEAM" program coordinate system goes along the socket line of the magnets and in the straight sections goes from magnet steel to magnet steel. The system used in laying out the positions of the magnets in TN 215 (called here "Layout"), which has not really been referred to as a coordinate system, also goes along the socket line of the magnets, but in the straight sections bends at the edge of the fringe field. This line is at a radius outside of the "BEAM" 1 ine and the steel to steel length along this line is a fraction of a mill longer than along the "BEAM" program 1ine. The Socket system consists of the lines connecting the magnet sockets. The OCO system is characterized by being displaced a distance $\mathrm{y}_{0}$ inward from the magnet socket line at the edge of the fringe field. Thus it is at a significantly smaller radius than any of the other systems. Table Al gives the lengths and angles for the various systems. Table 1 specifies $L_{i}, y_{0}$, and the angles. The other dimensions are calculated from the figure.

In Table Al for arithmetic purposes we assume very good precision. In particular we assume the lengths are accurate to five decimal places and the angles to four decimal places. Thus the fractions of a mill added to the effective lengths in the "Layout" system look ridiculous, but are essential to give consistency with the "BEAM" system and must be included in the calculations of TN 215 to give good agreement with the AGS standards book. The actual tolerances on the survey and the construction are of course much less severe.

Inside a magnet the OCO system is ill defined and probably not needed. However in Figure A2 we show a possible system. 
From a practical point of view, when we are dealing with straight sections between two similar magnets, we need only consider Figure A3 and Table A2, which show the OCO, the Socket, and the "BEAM" Program coordinate systems and the offsets between the different systems. These numbers are easily calculated from Fig. Al. For transition sections the geometry, while still trivial, is a little more complicated. For the transition from a long to a short magnet the layout is given by Figure $A 4$ where $\varepsilon$ is $2.14 \mathrm{mr}$. For the transition from a short to a long magnet the layout is given by Figure A5. This geometry has been incorporate into the calculation used to develop Table Al. Table A3 relates the type of straight section to the straight section number.

Figures $A 6$ and $A 7$ show the $0 C 0$ system in the "BEAM" system for straight sections where the transition from long to short or from short to long magnets is made. Table A4 gives a complete summary of the transformation from the "BEAM" program coordinate system to the OCO system.

Figure A8 attempts to clarify a subtle point of geometry. For the simple case shown in Fig. Al all the systems are parallel. However for the more complicated cases shown in Figures $\mathrm{A} 4$ and $\mathrm{A} 5$ the separation between the bend points matters, and it makes a difference if we are bending at the edge of the fringe field as in TN 215, or if we are bending at the edge of the magnet steel, as in the "BEAM" program coordinate system. From Figure A8:

$$
\begin{gathered}
\delta=\frac{\alpha_{L}-\alpha_{S}}{24} \\
=2.6806 \mathrm{mr} \\
\varepsilon=\frac{\left[\alpha_{L}-2 \tan \left(\theta_{L} / 2\right)\right]-\left[\alpha_{S}-2 \tan \left(\theta_{S} / 2\right)\right]}{28} \\
=2.1384 \mathrm{mr}
\end{gathered}
$$


The one half milliradian difference in the entrance and exit angles of the transition section shown in Table Al for "BEAM" and "Layout" results from the difference between $\varepsilon$ and $\delta$. In Appendix $V$ of TN 215 we said that due to the rounding down of the angle values the "BEAM" program did not close by 1.5 milliradian. A more correct statement is that due to the rounding down of the angle values the "BEAM" program would not close by 1.0 milliradians if it had not been fixed up by adding 0.02 milliradians to the calculated bend angle of 9.61 milliradians at the entrance to the short magnet wherever there is a transition between long and short magnets. Table AV-1 of TN 215 is confused as a result of some confusion on the part of the author. Table A5 is more usefu1. It shows the angles used in the "BEAM" program and a more exact set of values which are closer to the actual construction of the AGS. We believe that at some time these angles should be tried out in "BEAM".

THE PICK UP ELECTRODES IN THE OCO SYSTEM

The new pick up electrodes are located in the "BEAM" program coordinate system at (L. Ahrens, private communication):

$$
\begin{gathered}
y_{B}=0.00 \\
x_{B}=13.43 \text { inches }
\end{gathered}
$$

From the transformations given in Table A4, we can find that the beam position as read by the PUE's is given in the OCO system by the transforms of Table A6. We believe that at some time these transforms should be tried out in the PUE program.

\section{CIRCUMFERENCE}

From Table Al it is easy to calculate the sums of the chord lengths around the ring in the various systems. These are shown in Table A7. The actual path length of an orbit is somewhat greater since the path is curved in a magnet, adding about 0.003 inches per magnet, as is discussed in TN 215, Appendix $V$. 
Table Al

System Dimensions

System Magnet SS $\mathrm{L}_{\mathrm{e}} / 2$ Entrance $\theta / 2$ Magnet Exit $\theta / 2 \quad$ SS $\mathrm{L}_{\mathrm{e}} / 2$ No.

$\mathrm{L}$

inches $\mathrm{mr}$ inches inches $\quad \mathrm{mr}$

$\begin{array}{lll}94 & 13.9825 & 12.00020 \\ 94 & 13.9825 & 30.00020 \\ 94 & 16.6631 & 12.00018 \\ 79 & 11.7512 & 12.00014 \\ 79 & 11.7512 & 59.99549\end{array}$

"BEAM"

$\begin{array}{rr}6 & 32 \\ 7 & 14 \\ 8 & 32 \\ 9 & 14 \\ 10 & 14\end{array}$

Socket

6

9

10

OCO

$\begin{array}{rr}6 & 29.99714 \\ 7 & 11.99714 \\ 8 & 29.99714 \\ 9 & 11.99774 \\ 10 & 11.99832\end{array}$

13.98

13.98

13.98

9.63

11.75

34.99970

13.9825

13.9825

13.9825

10.1870

13.9825

13.9825

13.9825

11.7512

11.7512
11.7512
$90 \quad 13.98 \quad 14$

$90 \quad 13.98 \quad 32$

$\begin{array}{lll}75 & 11.75 \quad 14\end{array}$

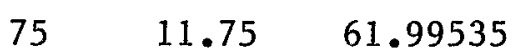

$84 \quad 13.9825 \quad 16.99970$

$84 \quad 13.9825 \quad 34.99975$

$84 \quad 15.5466 \quad 16.99975$

$\begin{array}{lll}69 & 11.7512 & 16.79980\end{array}$

$69 \quad 11.7512 \quad 64.99515$

$94 \quad 13.9825 \quad 11.99714$

$94 \quad 13.9825 \quad 29.99714$

$94 \quad 13.9825 \quad 11.99774$

$\begin{array}{lll}79 & 11.7512 & 11.99832\end{array}$

$\begin{array}{lll}79 & 11.7512 & 59.99367\end{array}$
$90 \quad 16.12 \quad 14$ 
Table A2

\section{Parameters for Fig. A2}

$$
\frac{a}{10^{-3} \text { inches }}
$$

5' SS

2' SS

between Long Magnets

$10^{\prime} \mathrm{SS}$

2' SS

between Short Magnets
42.0

42.0

35.2

35.2 $\frac{b}{10^{-3} \text { inches }}$

191.1

191.1

131.2

131.2 
Table A3

Classification of Straight Sections

\begin{abstract}
Type
Long to Long
\end{abstract}

Short to Short

Short to Long

Long to Short

\section{Length \\ feet}

2

5

2

10

2

2
SS No

$4,6,14,16$

$3,5,7,13,15,17$

$1,9,11,19$

10,20

2,12

8,18 
Table $\mathrm{A4}$

Transformations

If $\left(\mathrm{X}_{\mathrm{B}}, \mathrm{Y}_{\mathrm{B}}\right)$ are coordinates in the "BEAM" program coordinate system, then in the OCO system $\left(\mathrm{X}_{\mathrm{OCO}}, \mathrm{Y}_{\mathrm{OCO}}\right)$ are given by:

ELEMENT

Long Magnet

Short Magnet

5 Foot SS

10 Foot SS

2 Foot SS

(Long to Long)

2 Foot SS

(Short to Short)

2 Foot SS

(Long to Short)

2 Foot SS

(Short to Short)

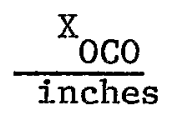

$\mathrm{X}_{\mathrm{B}}$

$\mathrm{X}_{\mathrm{B}}$

$x_{B}-2.0033$

$x_{B}-2.0014$

$x_{B}-2.0033$

$x_{B}-2.0014$

$x_{B}-2.0033$

$x_{B}-2.0014$
$\mathrm{Y}_{B}+0.1312$

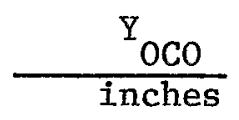

$\mathrm{Y}_{B}+0.2191$

$\mathrm{Y}_{\mathrm{B}}+0.1547$

$Y_{B}+0.1911$

$\mathrm{Y}_{\mathrm{B}}+0.1911$

$\mathrm{Y}_{\mathrm{B}}+0.1312$

$Y_{B}+0.1911-0.00214 X_{B}$

$Y_{B}+0.1312+0.00214 X_{B}$ 
Table A5

Magnet Entrance and Exit Angles

As built in

"Layout"

13.9825

11.7512

16.6631

9.0706
As built in

"BEAM" system

13.9825

11.7512

16.1209

9.6128
Used in

"BEAM" program

13.98

11.75

16.12

9.63 $\mathrm{mr}$

mr

Units

$\mathrm{mr}$

$\mathrm{mr}$ 
Table A6

Transformation for the PUE's from the "BEAM" to the OCO system

Straight Section

Long to Long

Long to Short

Short to Long $\underline{\text { SS No }}$

4,14

8, 18

2,12
$\mathrm{Y}_{\mathrm{OCO}}=\mathrm{Y}_{\mathrm{BEAM}}+$

0.191 inches

0.167 inches

0.156 inches 
Table A7

Circumference

Sum of Difference Effective radius

$\begin{array}{lccccc}\text { System } & \text { Chord Lengths } & \text { from BEAM } & \Sigma \mathrm{L} / 2 \pi & \text { Units } \\ \text { Layout } & 31,775.862 & +0.085 & 5,057.286 & \text { inches } \\ \text { BEAM } & 31,775.777 & 0 & 5,057.272 & \text { inches } \\ \text { Socket } & 31,775.669 & -0.108 & 5,057.255 & \text { inches } \\ \text { OCO } & 31,774.631 & -1.146 & 5,057.090 & \text { inches }\end{array}$




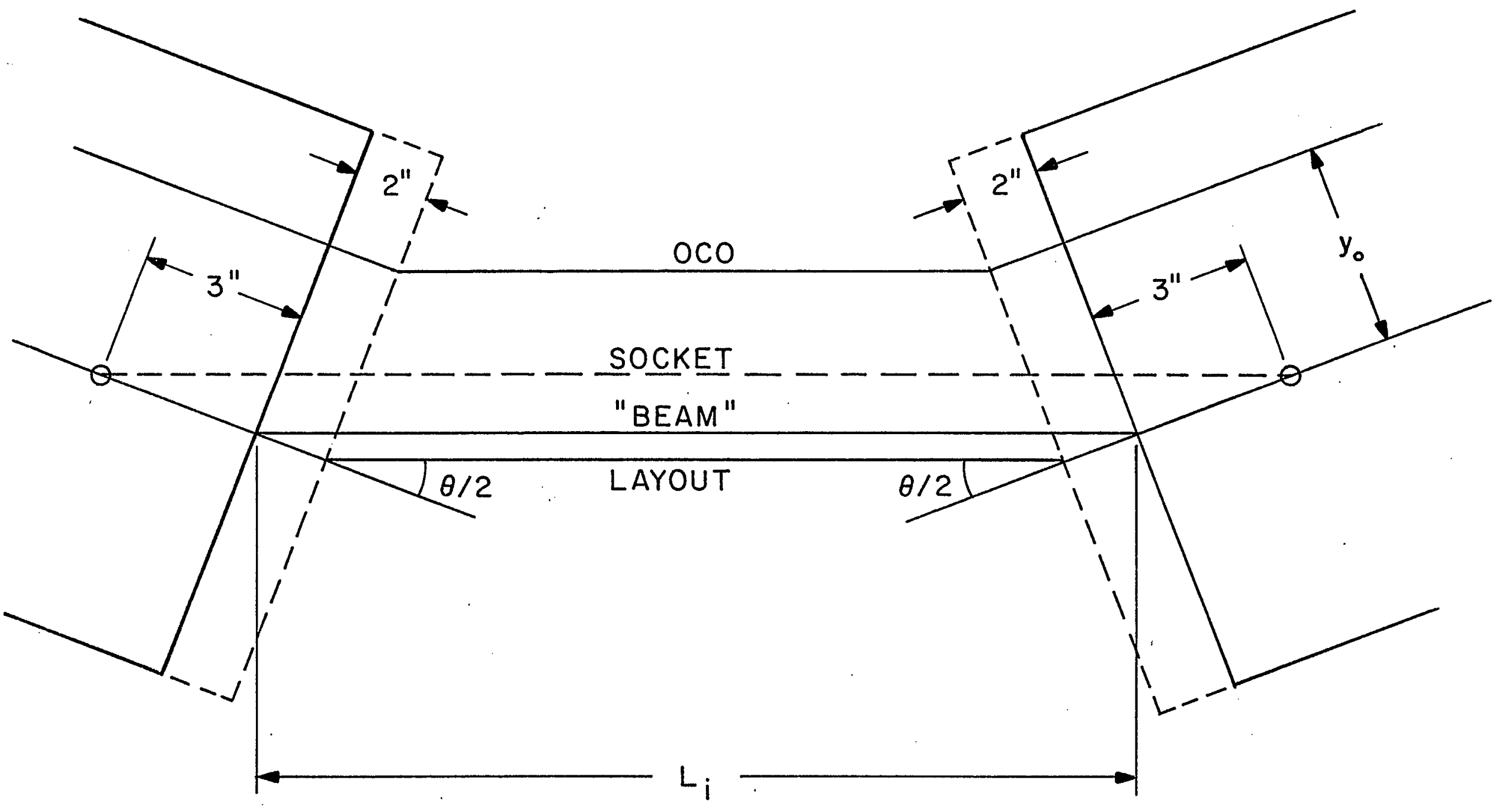




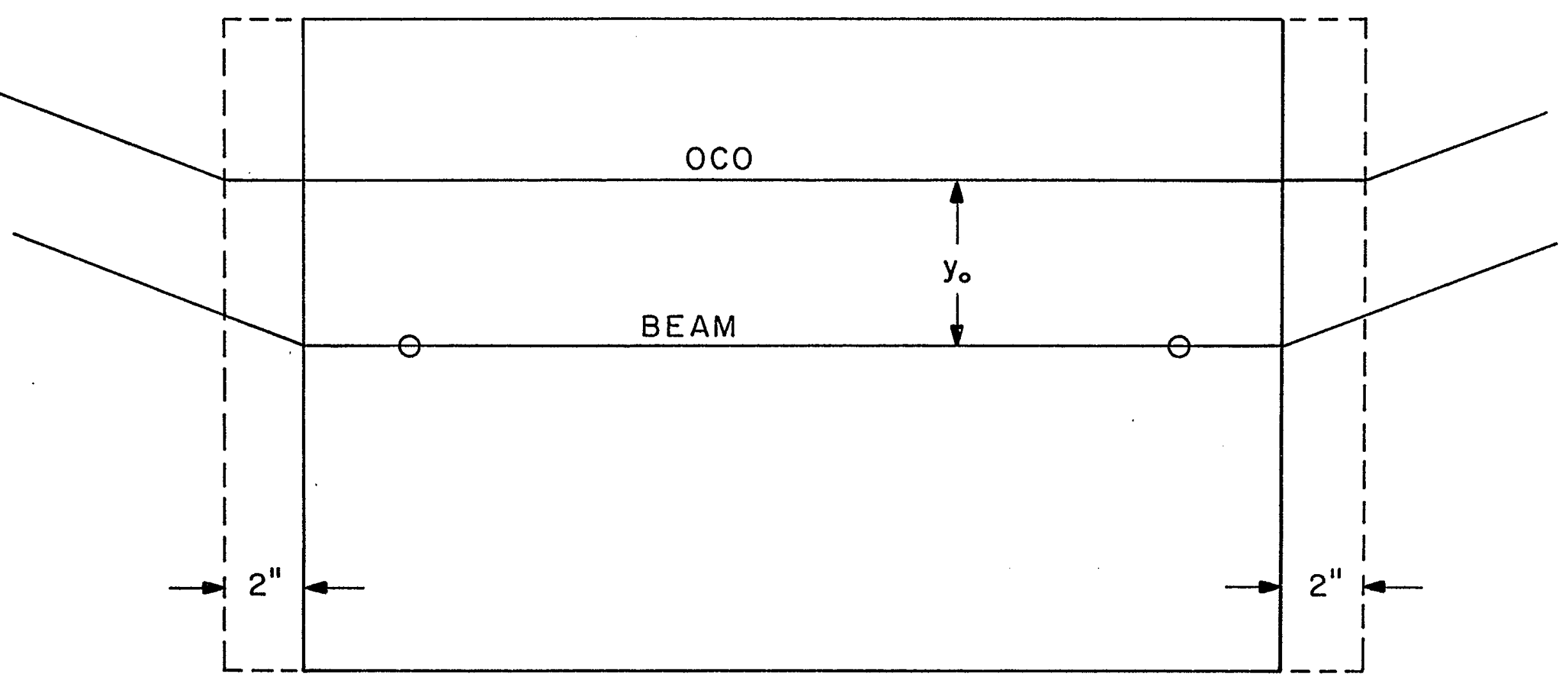

Figure A2 


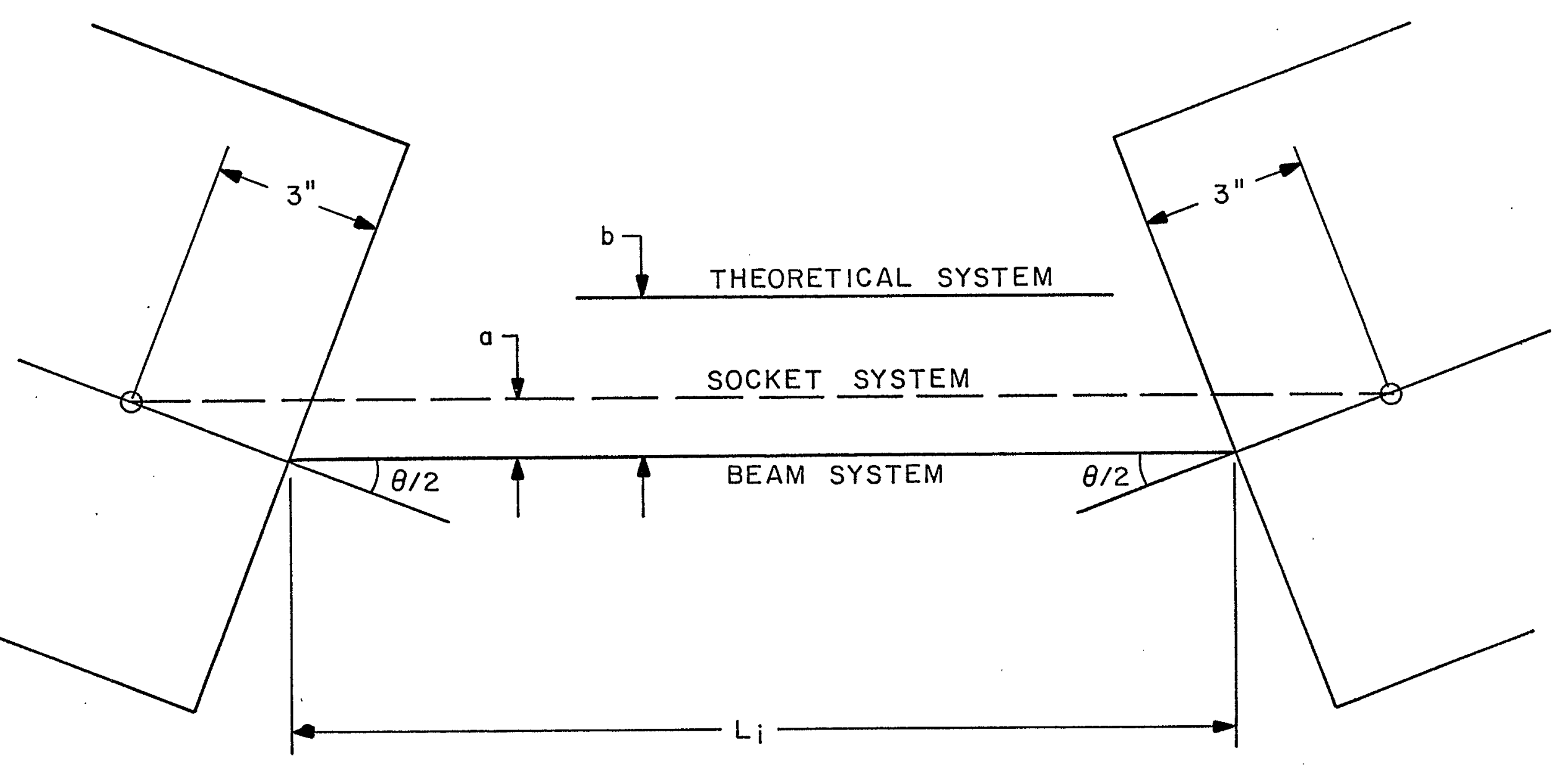

Figure A3 


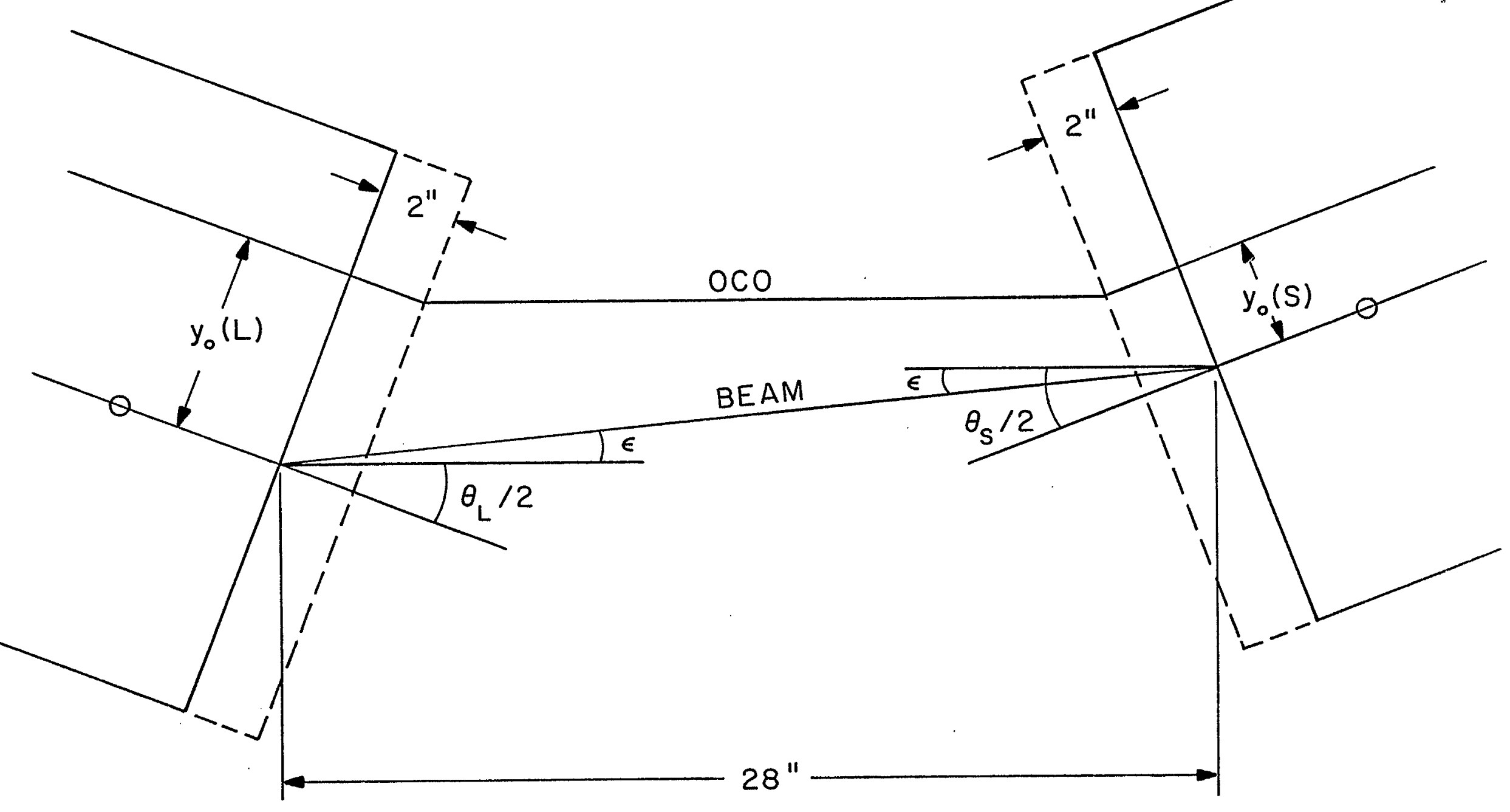




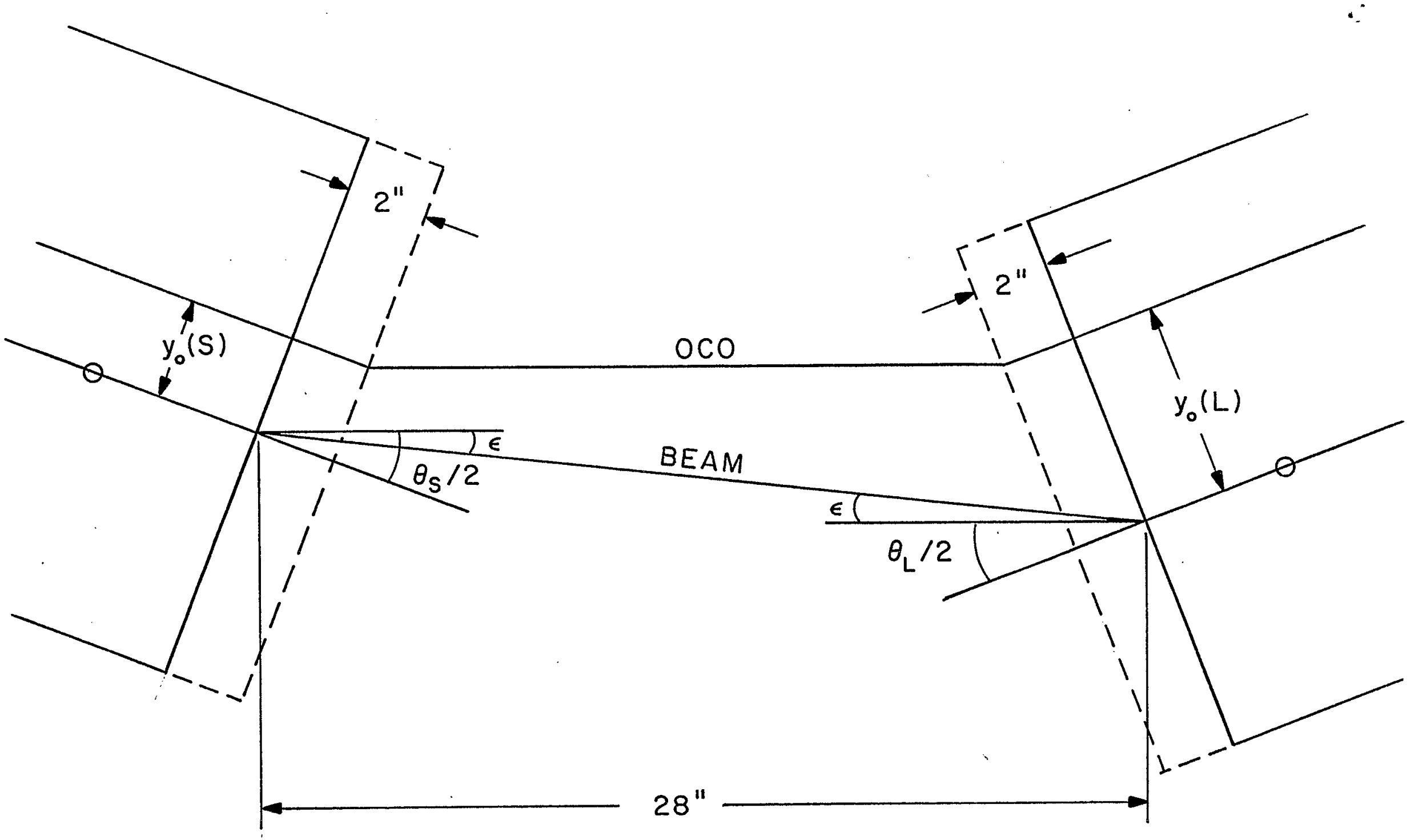




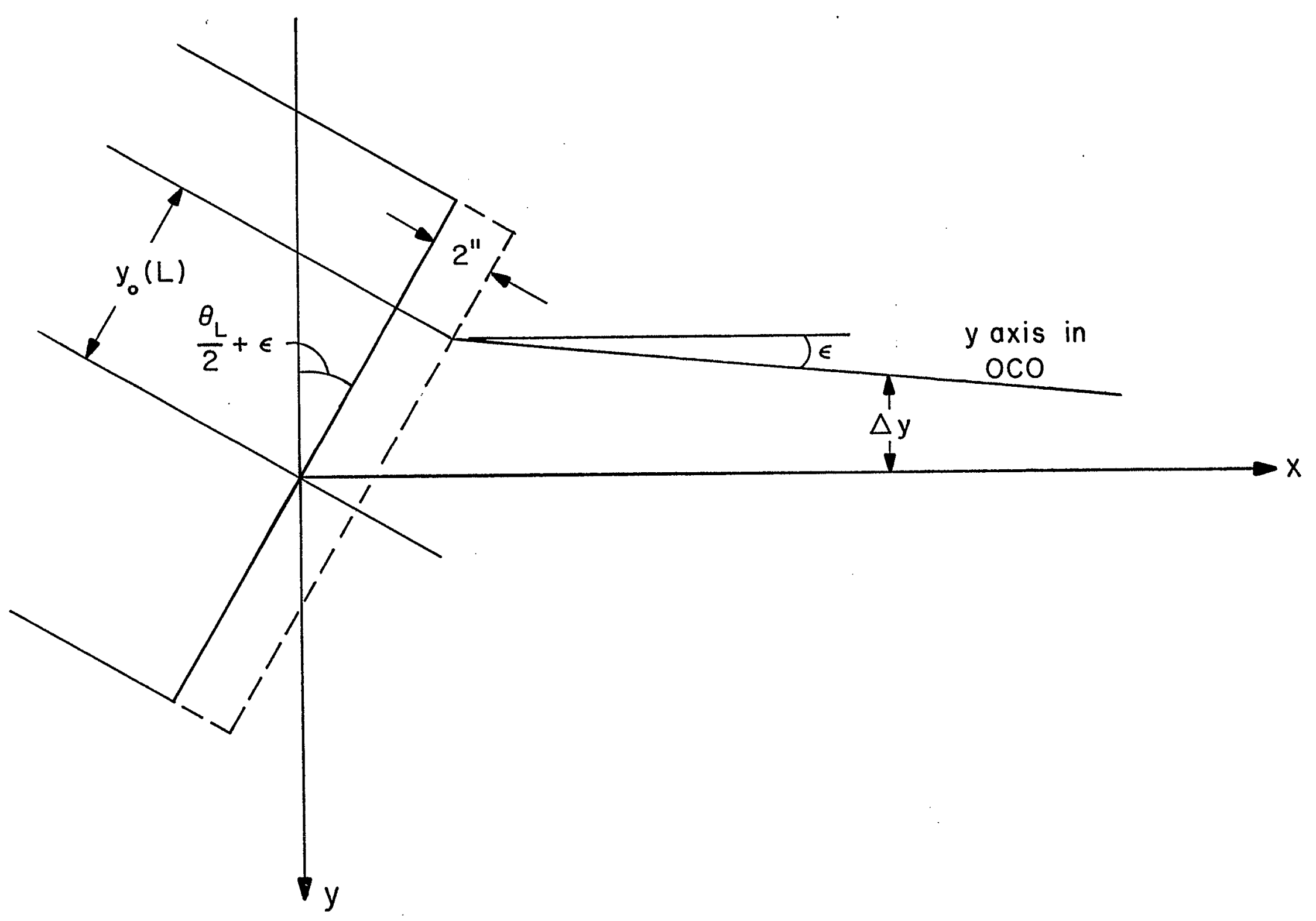




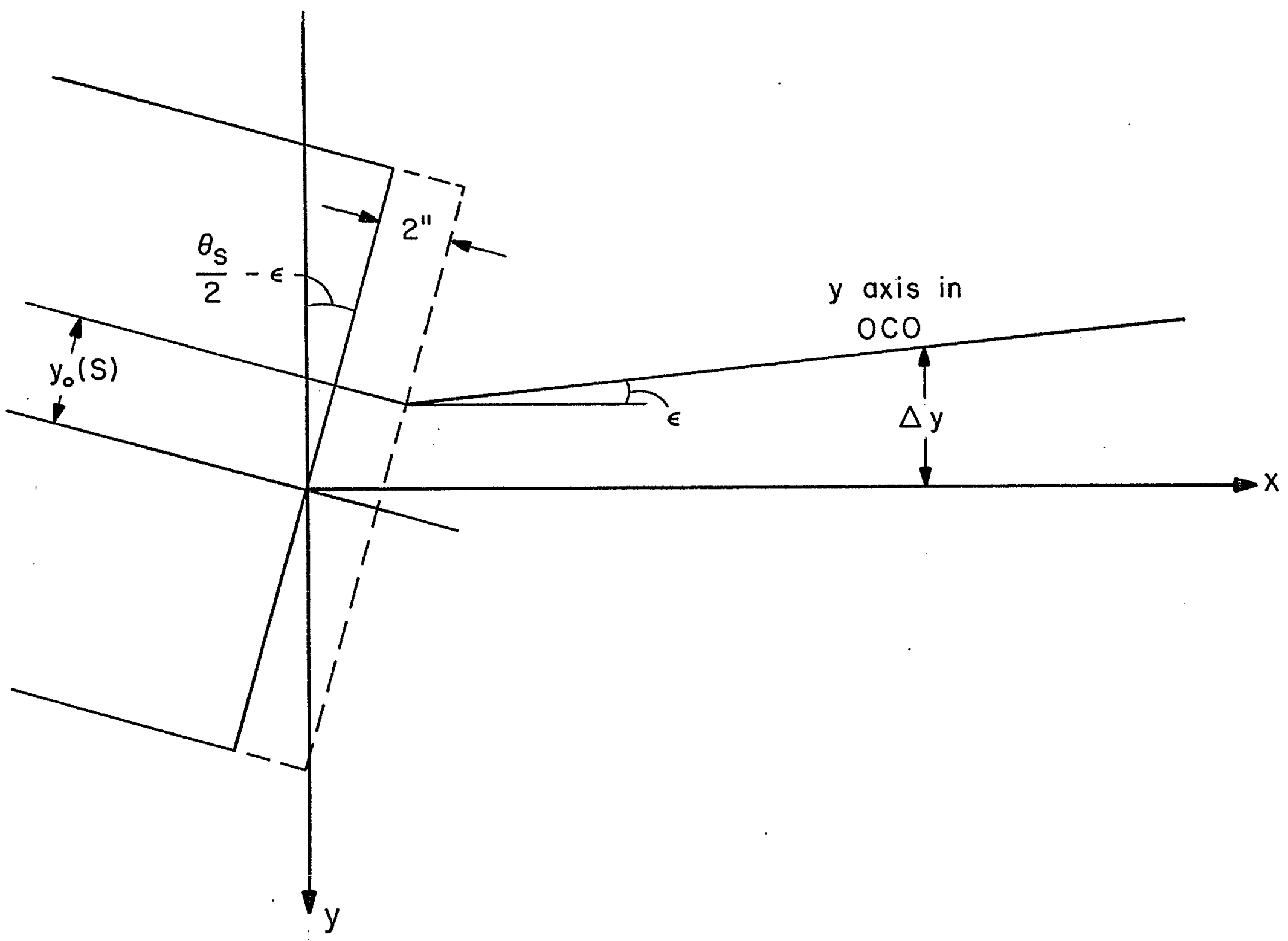

Figure A7 


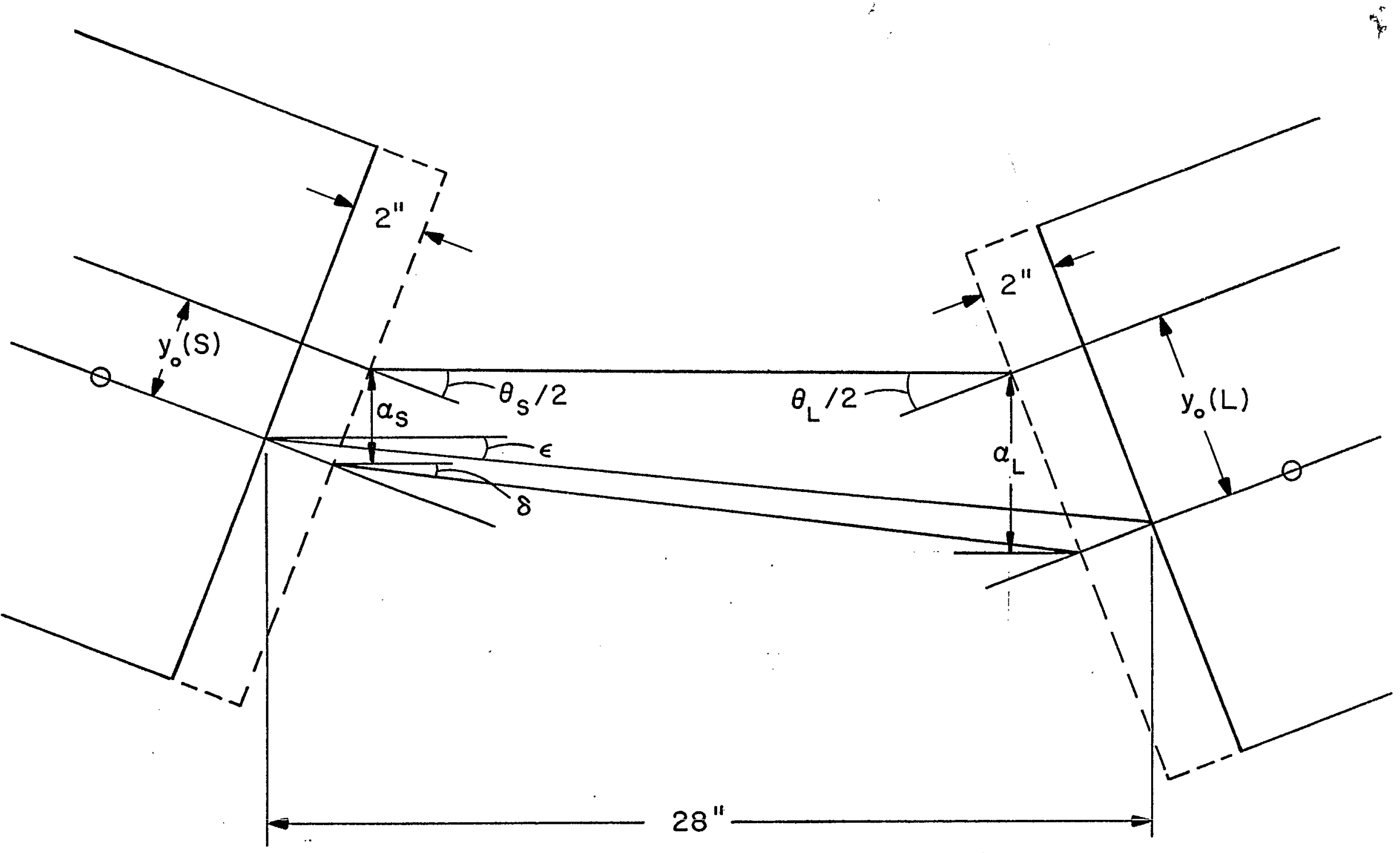

Figure A8 